\title{
HUMAN IDENTIFICATION USING FINGER IMAGES
}

\author{
Amruta P. Dharmadhikari ${ }^{1}$, S.R.Ganorkar ${ }^{2}$ \\ ${ }^{1}$ Dept. of E\&TC, Sinhgad College of Engineering, Vadgaon, Pune-41 \\ ${ }^{2}$ Dept. of E\&TC, Sinhgad College of Engineering, Vadgaon, Pune-41
}

\begin{abstract}
The system presented in this paper acquires the finger-vein and low resolution fingerprint images simultaneously. It combines its features using score level combination strategy.This paper presents approach in which it improves the performance of finger-vein identification systems. The minimum error distance classifier method has been used for score level combination.

Previous systems are referred to the decision level and feature level combination. These methods often suffer from the loss of information. In is quite complicated and largely responsible for the individuality of fingerprints and stead the score-level combination allows each of the observation to operate asynchronously, which is not possible in feature-level combinations.
\end{abstract}

Keywords-level-2 finger print features, personal identification, unconstrained imaging

\section{INTRODUCTION}

The structure of human fingers is complicated. Each person has unique skeleton. The anatomy of human fingers is largely responsible for individuality of fingerprints and finger veins. The individuality of fingerprints is refer to the random imperfections to the friction ridges and valleys. For level-2 fingerprint features, the images having resolution $400 \mathrm{dpi}$ are considered [5]. Features can be extracted from low resolution finger print images and those features are useful for fingerprint classification. These low resolution images illustrates friction creases and friction ridges but with varying clarity [12].

There are many options are available for online finger print identification, but very few are suitable for unconstrained finger images.In present paper the finger vein images acquired from near-infrared-based or thermal-infrared -based optical imaging.

The finger-vein patterns are extremely unique. The unique pattern of finger-vein can be observed in case of identical twins and even the different fingers of same person. There are two key features that are analyzed in support for the preference of finger-vein biometrics First, the finger veins are hidden structures; it is quite difficult to steal the finger-vein patterns of an individual, so it offers a quality of security. Second the use of finger-vein biometric offers strong anti spoofing capabilities as it can also ensure liveless in the presented fingers during the imaging. The finger-vein identification using random transform based statistical features and a probabilistic neural network classifier.

The curvelet-based extraction of finger-vein patterns and its classification using a back-propagation neural network are described in [13][14] have further improved the performance for the vein identification using a repeated line tracking algorithm.
The remainder of these paper is organized as follows. section 2 explains block diagram and its details. Section 3 presents the pre-processing of finger-vein image. Section 4 gives the feature extraction method for finger-vein and finger texture image. The matching of scores has been explained in section 5.Section 6 shows results and conclusion.

\section{BLOCK DIAGRAM}

The block diagram of the proposed system is shown in figure 1. Finger image from database has pick up for proposed framework.Image is then subjected to pre-processing with several steps. 1)Image normalization2)ROI extraction3)Image enhancement.The features of finger vein images are extracted using maximum curvature and repeated line tracking algorithm. Image is also subjected to morphological processing. Where features of finger texture images are extracted using Gabor filter.The minimum error distance classifier method has been used for score level combination. According to matching of scores it will shows the images processed belonging to which candidate.

Figure 2(a) and 2(b) shows the images from acquired database. These images are of left index finger. The higher scattering coeffient results in a darker appearance of finger-vein patterns 


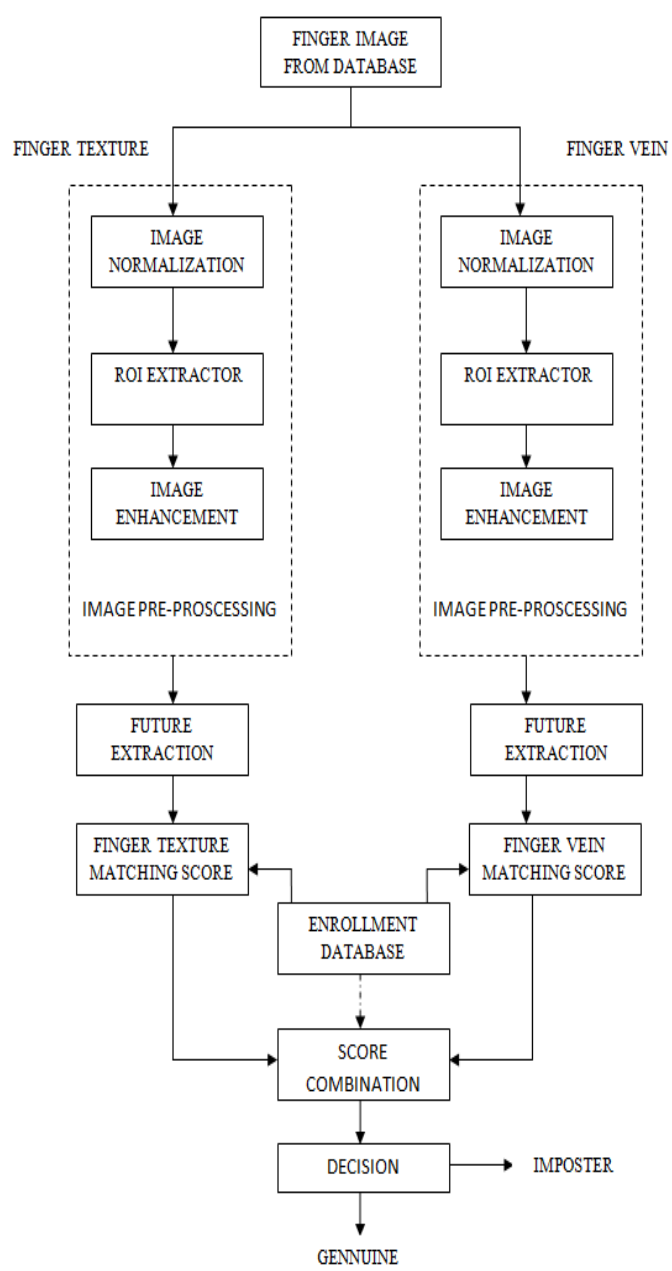

Fig.1 Block diagram of proposed system

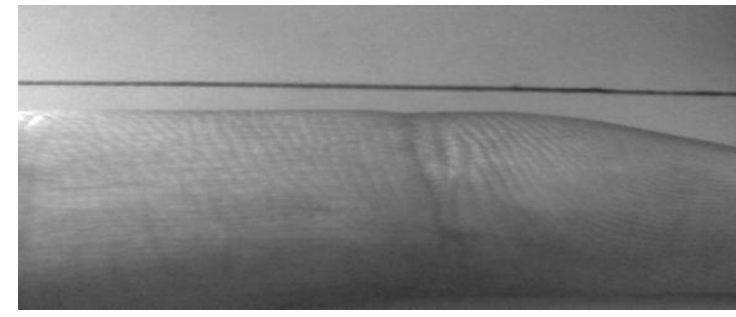

Fig 2(a) Finger texture sample from database

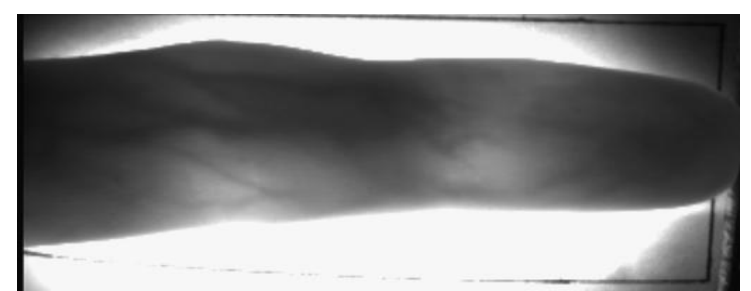

Fig 2(b) Finger vein sample from database

\section{IMAGE PREPROCESSING}

The captured finger vein images are contains rotational and translation variations so it is required to subject these images to pre-processing.

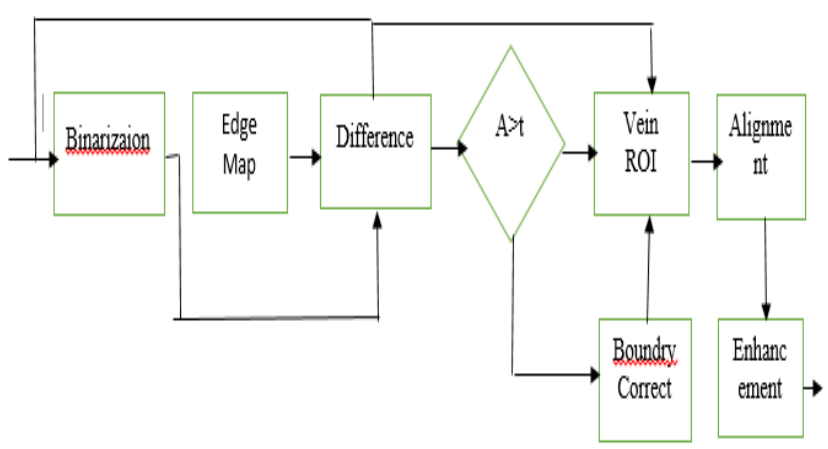

Fig. 3 Block diagram for pre-processing steps of vein images.A stands for area of thresholding

Each image is subjected to the binarization.For purpose we are using threshold value(t)230.It localizes the finger shape in image.Still after binarization background is appeared as connected to the bright region of finger. These is due to change in illumination pattern. Loosely connected regions are still appeared in resulting image.To eliminating these regions, Firstly sobel detector is applied to the entire image and edge map is subtracted from edge map.Still some blobs are appeared in image, to clear this blobs labelling and some morphological operations are performed on resulting image.Labelling is preferably used for blob extraction. In labelling connected components are uniquely labelled.It is part of ROI extraction, but it is not exactly as segmentation. It checks the connectivity of regions.Finger-vein images are not very clear due to uneven illumination of light and imperfect placement of fingers. Due to these images are subjected to the nonlinear image enhancement.

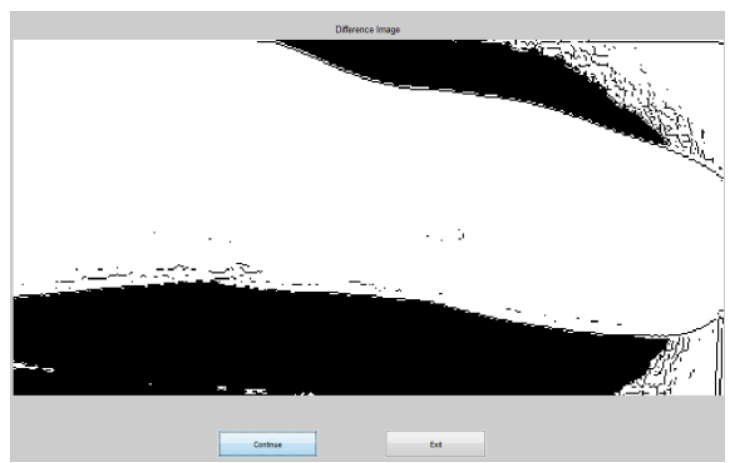

Fig. 4 Image before labeling

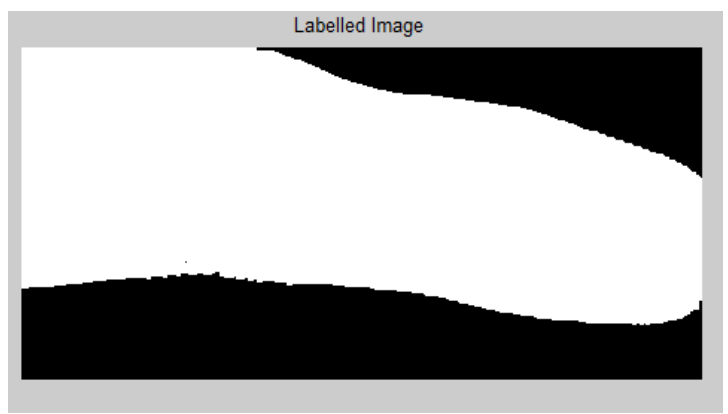

Fig.5 Labeled Image 
4. FEATURE EXTRACTION OF FINGERVEIN

\section{AND TEXTURE IMAGES}

\subsection{Finger Vein Feature Extraction}

In presented framework, features of vein images are get extracted by using maximum curvature and repeated line tracking algorithm. It gives the promising results. It traces the veins in the image by chosen directions according to predefined probability in the horizontal and vertical orientations. In case of repeated line tracking algorithm storing point(seed) has randomly selected. It repeats for certain number of times. As per name it locates the position that possesses the maximum curvature from the image profile. The profile are acquired in different direction; while all points are extracted, they are connected and combined according to the rules.

Conventional methods such as matched filters and morphological methods can extract patterns if the widths of veins are constant. However, these methods cannot extract veins that are narrower or wider than the assumed width, which degrades the accuracy of the personal identification. The repeated line tracking method can extract vein patterns from an unclear image but it cannot sufficiently extract thin veins because, number of times that the tracking point moves on thin veins tends to be small stastically.We propose a method that solves these problems by checking the curvature of the image profiles and emphasizing only the centerlines of veins. The center line are detected by searching for positions where the curvatures of a cross-sectional profile of a vein image are locally maximal.

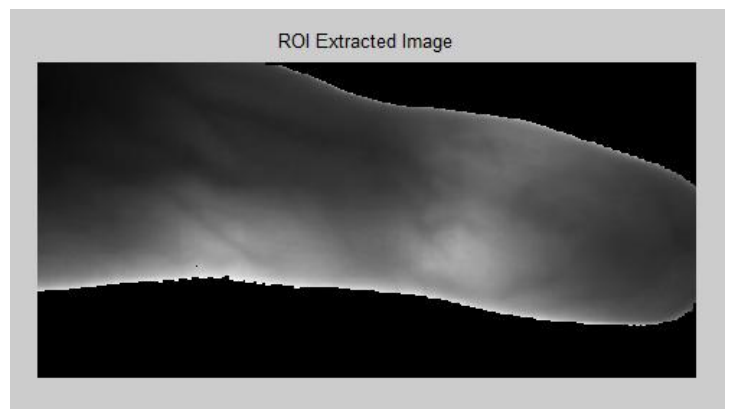

Fig.6 Feature extracted Image

\subsection{Finger Texture Feature Extraction}

The finger texture feature extraction has been done by Gabor filter. Frequency and orientation representation of Gabor filters are similar to those of human visual system and they have been found to be particularly appropriate for texture representation and discrimination. In the spatial domain a $2 \mathrm{D}$ gabor filter is a Guassian kernel function modulated by a sinusoidal plane wave .A set of Gabor filters with different frequency and orientations may be helpful for extracting useful features from an image.

Gabor filter are directly related to wavelets, since they can be designed for a number of dilations and rotations, However in general, explansion is not applied for gabor wavelets since this requires computation of bi-orthogonal wavelets, which may be very time consuming. Therefore usually a filter bank consisting of Gabor filters with various scales and rotations is created.

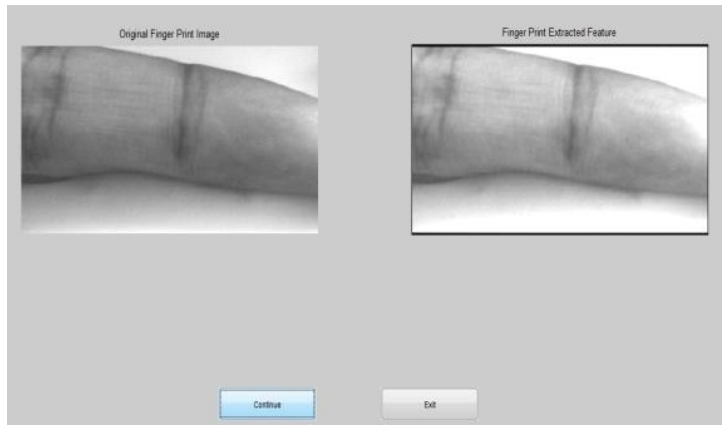

Fig.7 Feature extracted using Gabor filter.

\section{MATCHING SCORE}

After extracting the features, feature scores (testing samples) are created. In the method firstly it creates the testing set of all existing samples.i.e.the training dataset has been performed. I $n$ the proposed method the mean of each class and measure the distance to the unlabelled vector according to some metric. The class with a mean closest to the vector (with smallest distance) will be the label for that vector.

The first step is to compute, for each kind of label that found in the dataset, the mean of all sample vectors that were labeled with that particular label. Then, the distance between each unlabelled sample vector $\mathrm{x}$. We want to classify, and the means of all labelled sample vectors is minimized to give the class. If we would use the Euclidean distance metric for instance, we would find the class.

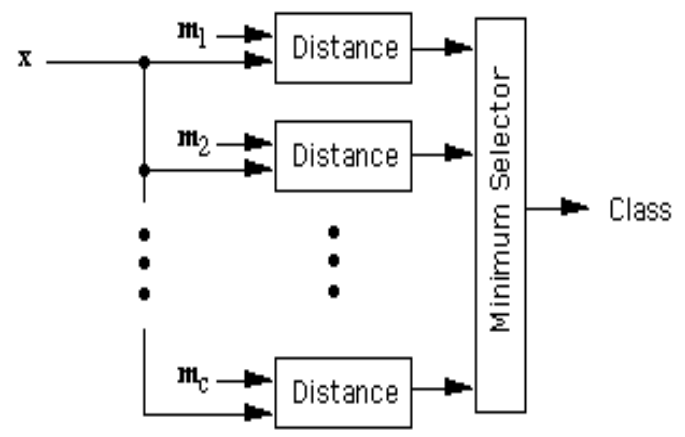

Fig 8 Minimum Distance classifier

Figure 8 shows schematic overview of the minimum distance procedure, where $\mathrm{m}$ stands for the mean of certain class. Where $\mathrm{m}$ stands for samples.

The class L can be given as follows:

$$
\mathrm{L}=\arg \min \sqrt{ } L=\arg \min \sqrt{\sum_{j=1}^{d}(X j-\mu j(L i)) 2}
$$

\section{RESULTS}

At the end, the implemented system of identification shows the image belongs to which candidate. It displays the image name. It has been showed in figure 9. 


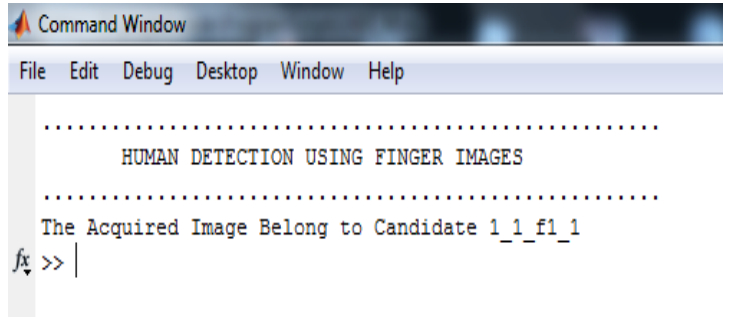

Fig.9 Final result displays candidate image name.

The database used contains 6264 images which includes finger vein \& finger texture image of each individual.

Maximum curvature \& repeated line tracking algorithm extract the desirable features from vein image. Pre-processing \& smoothing is performed by Gabor. It also extracts the finger texture features.

Minimum distance classifier gives the ultimate results.

\section{CONCLUSIONS}

The paper proposed and implemented the system in which the simultaneous use of two evidences i.e. finger vein pattern \& finger texture has taken place. Repeated line tracking algorithm performs the iterations due to which we get the exact \& clear vein pattern.

Gabor employs the frequency \& orientation representation. It extracts the texture features. Finger texture image is pre-processed and smoothened by Gabor.

Score level combination has been used for the proposed system. It generates and match the combined score. Block wise matching strategy has been employed for the system. Minimum distance method calculates the scores, it reduces the complexity \& consumed time.

\section{REFERENCES}

[1]. E. C. Lee and K. R. Park, "Restoration method of skin scattering blurred vein image for finger vein recognition," Electron. Lett., vol. 45, no. 21, pp. 1074-1076, Oct. 2009.

[2]. J-D.Wu and S.-H. Ye, "Driver identification using finger-vein patterns with Radon transform and neural network," Expert Sys. And Appl., vol. 36, no. 3, pp. 5793-5799, Apr. 2009

[3]. P. V. Reddy, A. Kumar, S. M. K. Rahman, and T. S. Mundra, "A new antispoofing approach for biometric devices," IEEE Trans. Biomed.Circuits Syst., vol. 2, no. 4, pp. 328-337, Dec. 2008.

[4]. N. Otsu, "A threshold selection method from gray-level histograms," IEEE Trans. Syst., Man, Cybern. B, Cybern., vol. 9, no. 1, pp. 62-66, Jan. 1979.

[5]. A.K. Jain, Y. Chen, and M. Demirkus, "Pores and ridges: High resolution fingerprint matching using level 3 features," IEEE Trans. PatternAnal. Mach. Intell., vol. 29, no. 1, pp. 15-27, Jan. 2007.

[6]. S. T. V. Parthasaradhi, R. Derakhshani, L. A. Hornak, and S. A. C. Schuckers, "Time-series detection of perspiration as a liveness test in fingerprint devices," IEEE Trans. Syst., Man, Cybern. C, Appl. Rev., vol. 35, no. 3, pp. 335-343, Aug. 2005. [7]. Antonelli, R. Capelli, D. Maio, and D. Maltoni, "Fake finger detection by skin distortion analysis," IEEE Trans. Inf. Forensics Security, vol. 1, no. 3, pp. 360-373, Sep. 2006.

[8]. K. Nandakumar, Y. Chen, S. C. Dass, and A. K. Jain, "Likelihood ratio based biometric score fusion," IEEE Trans. Pattern Anal.Mach. Intell., vol. 30, no. 2, pp. 342-347, Feb. 2008.

[9]. D.M. Weber and D. Casasent, "Quadratic Gabor filters for object detection," IEEE TransImage Process., vol. 10, no. 2, pp. 218-230, Feb. 2001.

[10]. T. Sim, S. Zhang, R. Janakiraman, and S. Kumar, "Continuous verification using multimodal biometrics," IEEE Trans. Pattern Anal.Mach.Intell., vol. 29, no. 4, pp. 687-700, Apr. 2007.

[11]. Handbook of remote biometrics,R.Chellappa,M. $\begin{array}{llll}\text { Tistarelli, } & \text { and } & \text { S.Z. } & \text { Li,Eds.New }\end{array}$ York:Springer-Verlag,Jul.2009.

[12]. Z. Zhang, S. Ma, and X. Han, "Multiscale feature extraction of fingervein patterns based on curvelets and local interconnection structure neural network," in Proc. ICPR, Hong Kong, 2006, pp. 145-148.

[13]. N. Miura,A. Nagasaka, and T. Miyatake, "Feature extraction of fingervein patterns based on repeated line tracking and its application to personal identification,"Mach. Vis. Appl., vol. 15, no. 4, pp. 194-203, Oct.2004.

[14]. J. Mobley and T. Vo-Dinh, Biomedical Photonics Handbook. Boca Raton, FL: CRC Press, 2003. 